\title{
Theoretical Electronic and Rovibrational Studies for Anions of Interest to the DIBs
}

\author{
R. C. Fortenberry $\dagger$ \\ NASA Ames Research Center, Moffet Field, CA 94035, USA \\ email: ryan.c.fortenberry@nasa.gov
}

\begin{abstract}
The dipole-bound excited state of the methylene nitrile anion $\left(\mathrm{CH}_{2} \mathrm{CN}^{-}\right)$has been suggested as a candidate carrier for a diffuse interstellar band (DIB) at $803.8 \mathrm{~nm}$. Its corresponding radical has been detected in the interstellar medium (ISM), making the existence for the anion possible. This work applies state-of-the-art ab initio methods such as coupled cluster theory to reproduce accurately the electronic excitations for $\mathrm{CH}_{2} \mathrm{CN}^{-}$and the similar methylene enolate anion, $\mathrm{CH}_{2} \mathrm{CHO}^{-}$. This same approach has been employed to indicate that 19 other anions may possess electronically excited states, five of which are valence in nature. Concurrently, in order to assist in the detection of these anions in the ISM, work has also been directed towards predicting vibrational frequencies and spectroscopic constants for these anions through the use of quartic force fields (QFFs). Theoretical rovibrational work on anions has thus far included studies of $\mathrm{CH}_{2} \mathrm{CN}^{-}, \mathrm{C}_{3} \mathrm{H}^{-}$, and is currently ongoing for similar systems.
\end{abstract}

Keywords. astrochemistry, molecular data, ISM: molecules, infrared: ISM, radio lines: ISM, ultraviolet: ISM

\section{Introduction}

Small molecular anions have been proposed as possible carriers of the diffuse interstellar bands (DIBs) for over a decade now with the initial observation of a coincidence between several bands of the open-shell $\mathrm{C}_{7}^{-}$radical anion and at least five DIBs observed in Tulej et al. (1998). Unfortunately, the spectral features and, to a greater extent, the spectral shapes varied too greatly for this anion to be considered a DIB carrier from the work by McCall et al. (2001). Later, the dipole-bound excited state of $\mathrm{CH}_{2} \mathrm{CN}^{-}$has been noted by Sarre (2000) and, later, by Cordiner \& Sarre (2007) to possess an excitation energy very close to a DIB at $8038 \AA$. This hypothesis has yet to be fully explored and opens a new path by which DIB carriers may be explored.

A dipole-bound state of an anion is similar to a Rydberg state of a neutral. However, a Rydberg state is bound by the interaction of the diffuse electron with the now positively charged core of the molecule. Hence, a monopole-monopole interaction results where the core of the molecule functions as a hydrogen nucleus. Dipole-bound states of anions do not have the benefit of such an interaction since removal of the electron from the anion results in a neutral molecular core. As such, most anionic electrons are weakly bound if at all. However, if the neutral core of the molecule, treated as an independent system, has a large enough dipole moment, the electron may remain bound within the system (see Simons et al. 2008). The minimum dipole moment necessary for a neutral molecule to bind an extra electron in a $\sigma$-type orbital is $1.625 \mathrm{D}$ as originally derived by Fermi \& Teller (1947). Physical conditions actually place this threshold at more than $2.5 \mathrm{D}$ as detailed by Gutsev \& Adamowicz (1995). Since these dipole-bound states, like Rydberg

$\dagger$ Present address: Department of Chemistry, Georgia Southern University, Statesboro, GA 30460, USA 
states, exist on the cusp of the dissociation continuum, only one such state can be present for a given anion unless the dipole moment is closer to $10 \mathrm{D}$. As a result, the electronic spectra of anions are much simpler than cations or neutral molecules.

$\mathrm{CH}_{2} \mathrm{CN}^{-}$is a closed-shell, valence anion in its $\tilde{X}^{1} A^{\prime}$ state. As a result, electronic excitation is possible into a dipole-bound excited state and is known to exist experimentally at $1.543 \mathrm{eV}(803.8 \mathrm{~nm})$ from Lykke et al. (1987). $\mathrm{CH}_{2} \mathrm{CHO}^{-}$is also a known closedshell valence ground state anion and to possess a dipole-bound excited state at $1.759 \mathrm{eV}$ (704.9 nm) from Mullin et al. (1992). Additionally, the $\mathrm{CH}_{2} \mathrm{CN}$ radical has been observed in the interstellar medium (ISM), making the existence of $\mathrm{CH}_{2} \mathrm{CN}^{-}$well within reason. With these anions as benchmarks, high-level, ab initio, quantum chemical computations are employed to explore the electronic spectra of several small anions.

\section{Computational Methods}

As outlined previously by Fortenberry \& Crawford (2011a,b) and Fortenberry (2013), energy differences from geometry optimizations of the ground states for the anion and neutral radical with $\mathrm{CCSD}(\mathrm{T}) /$ aug-cc-pVTZ give adiabatic electron binding energies (eBEs), which is the energy necessary to remove the electron from the system. EOMCCSD/d-aug-cc-pVDZ geometery optimizations of the ground and first excited states of the anion produce adiabatic excitation energies from the energy differences of these optimized geometries. Even though this is a best-to-best comparison that should prove more accurate, geometry optimization is the manipulation of several variables. As a result, vertical computations are also employed which freeze the geometry at a select point (in this case the $\operatorname{CCSD}(\mathrm{T}) /$ aug-cc-pVTZ geometry for the ground state of the anion) and manipulate the wavefunction either for electron removal or excitation. The vertical excitation energies are computed with EOM-CCSD with an increasing level of diffuseness in the spatial extent of the cc-pVDZ and cc-pVTZ basis sets and are derived in an even-tempered fashion. Convergence to an excitation energy only until highly diffuse basis sets are used implies that the electron probability density is further away from the core of the molecule. This indicates, for the present purposes, the presence of a dipolebound excited state.

Additionally, highly-accurate rovibrational computations are employed on a selection of anions that possess electronically excited states. The spectroscopic constants and vibrational frequencies provide more data to assist in their interstellar detection. This procedure involving quartic force fields (QFFs), which is a numerical solving of the nuclear potential energy function as a fourth-order Taylor series expansion, is detailed elsewhere: Huang \& Lee (2008) and Fortenberry et al. (2011). Accuracies for computed spectroscopic data compared with experiment for some closed-shell systems are known to be better than $0.1 \%$ as is the case with $\mathrm{C}_{3} \mathrm{H}_{3}{ }^{+}$reported by Huang et al. (2011); $\mathrm{HOCO}^{+}$ from Fortenberry et al. (2012); and $\mathrm{NNOH}^{+}$given in Huang et al. (2013a).

\section{Discussion}

As shown in Table 1, the adiabatic eBE computations place the $\mathrm{CH}_{2} \mathrm{CN}^{-}$at $1.48 \mathrm{eV}, 0.06 \mathrm{eV}$ less than the experimental value, while the adiabatic $\mathrm{CH}_{2} \mathrm{CHO}^{-} \mathrm{eBE}$ is computed to be $1.77 \mathrm{eV}$ or $0.05 \mathrm{eV}$ less than experiment. The adiabatic first excited state energy for $\mathrm{CH}_{2} \mathrm{CN}^{-}$is $1.49 \mathrm{eV}, 0.04 \mathrm{eV}$ less than experiment. The same value for $\mathrm{CH}_{2} \mathrm{CHO}^{-}$is $1.77 \mathrm{eV}$ or $0.01 \mathrm{eV}$ more than experiment. Electronic transitions within $0.1 \mathrm{eV}$ of experiment are the best that can be expected even for the EOM-CCSD method, and these computations for the benchmarking anions highlight the level of accuracy expected. 
Table 1. Computed dipole moments (in Debye for the corresponding neutral radical), eBEs (in $\mathrm{eV})$, and first adiabatic excited state transition energies $(\mathrm{eV})$ and wavelengths $(\mathrm{nm})$ for several anions.

\begin{tabular}{|c|c|c|c|c|c|}
\hline Molecule & | Dipole | & $\mathrm{eBE}$ & Transition & Energy & $\lambda$ \\
\hline $\mathrm{CH}_{2} \mathrm{CN}^{-}$ & 3.509 & 1.48 & $1^{1} B_{1} \leftarrow 1^{1} A^{\prime}$ & 1.49 & 830 \\
\hline $\mathrm{CH}_{2} \mathrm{SiN}^{-}$ & 4.110 & 2.49 & $1^{1} B_{1} \leftarrow 1{ }^{1} A_{1}$ & 2.10 & 590 \\
\hline $\mathrm{SiH}_{2} \mathrm{CN}^{-}$ & 3.524 & 2.31 & $2{ }^{1} A^{\prime} \leftarrow 1^{1} A^{\prime}$ & 2.39 & 519 \\
\hline $\mathrm{CH}_{2} \mathrm{CHO}^{-}$ & 2.921 & 1.77 & $1^{1} A^{\prime \prime} \leftarrow 1^{1} A^{\prime}$ & 1.77 & 702 \\
\hline $\mathrm{CH}_{2} \mathrm{SiHO}^{-}$ & 4.452 & 2.45 & $1^{1} A^{\prime \prime} \leftarrow 1^{1} A^{\prime}$ & 2.47 & 503 \\
\hline $\mathrm{SiH}_{2} \mathrm{CHO}^{-}$ & 2.391 & 1.93 & $2^{1} A \leftarrow 1^{1} A$ & 2.20 & 564 \\
\hline $\mathrm{CH}_{2} \mathrm{ON}^{-}$ & 2.335 & $0.46^{a}$ & $2^{1} A \leftarrow 1^{1} A$ & 0.89 & 1396 \\
\hline $\mathrm{CH}_{2} \mathrm{SN}^{-}$ & 2.703 & 1.98 & $2^{1} A^{\prime} \leftarrow 1^{1} A^{\prime}$ & 1.95 & 634 \\
\hline $\mathrm{CH}_{2} \mathrm{NO}^{-}$ & 2.317 & 1.42 & $2{ }^{1} A^{\prime} \leftarrow 1^{1} A^{\prime}$ & 1.55 & 799 \\
\hline $\mathrm{CH}_{2} \mathrm{PO}^{-}$ & 2.477 & 2.82 & $1^{1} A^{\prime \prime} \leftarrow 1{ }^{1} A^{\prime}$ & 2.89 & 429 \\
\hline $\mathrm{C}_{3} \mathrm{~N}^{-}$ & 2.889 & 4.38 & $2{ }^{1} \Sigma^{+} \leftarrow 1{ }^{1} \Sigma^{+}$ & 4.71 & 263 \\
\hline $\mathrm{SiCCN}^{-}$ & 4.010 & 3.44 & $2{ }^{1} A^{\prime} \leftarrow 1{ }^{1} \Sigma^{+}$ & 3.13 & 397 \\
\hline $\mathrm{CCSiN}^{-}$ & $0.672^{b}$ & 3.93 & $1^{1} A^{\prime \prime} \leftarrow 1{ }^{1} \Sigma^{+}$ & 2.49 & 498 \\
\hline $\mathrm{SiCN}^{-}$ & 3.188 & 1.27 & $1{ }^{1} \Pi \leftarrow 1{ }^{1} \Sigma^{+}$ & 1.37 & 906 \\
\hline $\mathrm{SiNC}^{-}$ & 2.709 & 0.92 & $1^{1} \Pi \leftarrow 1{ }^{1} \Sigma^{+}$ & 1.07 & 1158 \\
\hline $\mathrm{CN}^{-}$ & 1.471 & 3.82 & $1^{1} \Pi \leftarrow 1^{1} \Sigma^{+}$ & 4.43 & 280 \\
\hline $\mathrm{SiN}^{-}$ & 2.585 & 2.97 & $2{ }^{1} \Sigma^{+} \leftarrow 1{ }^{1} \Sigma^{+}$ & 3.23 & 383 \\
\hline $\mathrm{C}_{2} \mathrm{~F}^{-}$ & 1.075 & 3.17 & $2{ }^{1} A^{\prime} \leftarrow 1^{1} \Sigma^{+}$ & 3.51 & 354 \\
\hline $\mathrm{HCCO}^{-}$ & 2.170 & 2.39 & $1^{1} A^{\prime \prime} \leftarrow 1^{1} A^{\prime}$ & 2.37 & 523 \\
\hline $\mathrm{CCOH}^{-}$ & 4.401 & 2.52 & $1^{1} A^{\prime \prime} \leftarrow 1^{1} A^{\prime}$ & 2.43 & 511 \\
\hline $\mathrm{CCSH}^{-}$ & 4.492 & 2.86 & $1^{1} A^{\prime \prime} \leftarrow 1^{1} A^{\prime}$ & $2.82^{c}$ & 440 \\
\hline $\mathrm{C}_{3} \mathrm{H}^{-}$ & 3.409 & 1.83 & $1^{1} A^{\prime \prime} \leftarrow 1^{1} A^{\prime}$ & 0.93 & 1328 \\
\hline $\mathrm{CCSiH}^{-}$ & $\sim 3^{c}$ & 3.11 & $1^{1} A^{\prime \prime} \leftarrow 1^{1} A^{\prime}$ & 2.06 & 602 \\
\hline $\mathrm{CCNH}_{2}^{-}$ & 5.903 & 1.97 & $1^{1} B_{1} \leftarrow 1^{1} A^{\prime}$ & 2.00 & 620 \\
\hline $\mathrm{CCPH}_{2}^{-}$ & 4.759 & 3.21 & $2{ }^{1} A^{\prime} \leftarrow 1^{1} A^{\prime}$ & 3.16 & 392 \\
\hline $\mathrm{BH}_{3} \mathrm{NH}_{2}^{-}$ & 2.524 & 2.28 & $2^{1} A^{\prime} \leftarrow 1^{1} A^{\prime}$ & 2.53 & 490 \\
\hline $\mathrm{BH}_{3} \mathrm{PH}_{2}^{-}$ & 2.889 & 2.74 & $2^{1} A^{\prime} \leftarrow 1^{1} A^{\prime}$ & 2.78 & 446 \\
\hline $\mathrm{AlH}_{3} \mathrm{NH}_{2}^{-}$ & 2.579 & 3.22 & $2^{1} A^{\prime} \leftarrow 1^{1} A^{\prime}$ & 3.17 & 391 \\
\hline $\mathrm{AlH}_{3} \mathrm{PH}_{2}^{-}$ & 2.167 & 3.25 & $2^{1} A^{\prime} \leftarrow 1^{1} A^{\prime}$ & 3.21 & 387 \\
\hline
\end{tabular}

${ }^{a}$ This value is from the non-minimum geometry but functions as an upper bound estimate.

${ }^{b}$ The lowest energy isomer of the radical is a cyclic ring with the $\mathrm{N}$ bonded to the $\mathrm{Si}$. The linear isomer has a larger dipole moment, but its stability is uncertain.

${ }^{c}$ These values are upper- $\left(\mathrm{CCSH}^{-}\right)$or lower- $\left(\mathrm{CCSiH}^{-}\right)$bound estimates.

Theoretical and computational chemistry are uniquely suited to explore properties of various molecules where laboratory examination can prove quite difficult. Hence, knowing the accuracies of this approach, new anions can be explored for their excited state properties. The anions must be closed-shell, valence anions in order to possess a higher electronic state. The corresponding neutral radicals must have large dipole moments of greater than at least 2 D. Small anions are preferrable for the methods required and for the astrophysical conditions expected. The list of anions explored to date along with their computed properties is given in Table 1 and is a compilation of data from the studies undertaken by Fortenberry \& Crawford (2011a,b) and Fortenberry (2013). Of those anions listed, 19 new ones clearly have adiabatic excitation energies that are less than or no more than $0.1 \mathrm{eV}$ above the eBEs. These include $\mathrm{CH}_{2} \mathrm{SiN}^{-}, \mathrm{SiH}_{2} \mathrm{CN}^{-}$, $\mathrm{CH}_{2} \mathrm{SiHO}^{-}, \mathrm{SiN}^{-}, \mathrm{CCOH}^{-}, \mathrm{HCCO}^{-}, \mathrm{SiCCN}^{-}, \mathrm{CSiCN}^{-}, \mathrm{CCSiN}^{-}, \mathrm{SiNC}^{-}, \mathrm{CH}_{2} \mathrm{SN}^{-}$, $\mathrm{C}_{3} \mathrm{H}^{-}, \mathrm{CCSiH}^{-}, \mathrm{CCSH}^{-}, \mathrm{CCNH}_{2}^{-}, \mathrm{CCPH}_{2}^{-}, \mathrm{BH}_{3} \mathrm{PH}_{2}^{-}, \mathrm{AlH}_{3} \mathrm{NH}_{2}^{-}$, and $\mathrm{AlH}_{3} \mathrm{PH}_{2}^{-}$. Most 
Table 2. EOM-CCSD vertical excitation energies (in eV), oscillator strengths, ${ }^{a}$ and vertical electron binding energies (in $\mathrm{eV}$ ) from ground state $\operatorname{CCSD}(\mathrm{T}) /$ aug-cc-pVTZ geometries for several basis sets. ${ }^{b}$

\begin{tabular}{|c|c|c|c|c|c|c|c|c|c|}
\hline Molecule Transition & \multicolumn{7}{|c|}{$\mid$ pVDZ apVDZ dapVDZ tapVDZ|pVTZapVTZ dapVTZ| } & \multicolumn{2}{|c|}{$f \quad \| \mathrm{eBE}^{c}$} \\
\hline $\mathrm{CH}_{2} \mathrm{SiN}^{-} 1{ }^{1} B_{1} \leftarrow 1{ }^{1} A_{1}$ & 2.90 & 2.24 & 2.12 & 2.12 & 2.69 & 2.29 & 2.23 & $3 \times 10^{-3}$ & \multirow{2}{*}{$\begin{array}{l}3 \\
3\end{array}$} \\
\hline $2{ }^{1} B_{1} \leftarrow 1{ }^{1} A_{1}$ & 6.44 & 3.38 & 2.59 & 2.39 & 5.87 & 3.33 & 2.73 & $4 \times 10^{-3}$ & \\
\hline $\mathrm{SiCCN}^{-} 2^{1} \Sigma^{+} \leftarrow 1{ }^{1} \Sigma^{+}$ & 3.58 & 3.30 & 3.27 & 3.27 & 3.41 & 3.24 & 3.23 & $1 \times 10^{-5}$ & \multirow{2}{*}{\begin{tabular}{l|l}
5 & 3.33 \\
2 & 3.33
\end{tabular}} \\
\hline $1{ }^{1} \Pi \leftarrow 1{ }^{1} \Sigma^{+}$ & 7.17 & 4.50 & 3.50 & 3.36 & 6.45 & 4.36 & 3.62 & $1 \times 10^{-2}$ & \\
\hline $\mathrm{CCSiN}^{-} 1^{1} \Pi \leftarrow 1^{1} \Sigma^{+}$ & 4.15 & 3.75 & 3.71 & 3.71 & 3.89 & 3.71 & 3.70 & $5 \times 10^{-2}$ & \multirow{3}{*}{4.51} \\
\hline $2^{1} \Sigma^{+} \leftarrow 1^{1} \Sigma^{+}$ & 4.35 & 4.22 & 4.21 & 4.21 & 4.23 & 4.17 & 4.16 & $1 \times 10^{-7}$ & \\
\hline $2{ }^{1} \Pi \leftarrow 1{ }^{1} \Sigma^{+}$ & 6.20 & 5.71 & 4.82 & 4.60 & 5.99 & 5.68 & 4.93 & $2 \times 10^{-5}$ & \\
\hline \multirow{2}{*}{$\mathrm{C}_{3} \mathrm{H}^{-}$} & 1.21 & 1.15 & 1.14 & 1.14 & 1.17 & 1.14 & 1.13 & $2 \times 10^{-3}$ & \multirow{2}{*}{2.34} \\
\hline & 6.38 & 3.01 & 2.46 & 2.37 & 5.68 & 3.03 & 2.61 & $3 \times 10^{-3}$ & \\
\hline \multirow[t]{2}{*}{$\mathrm{CCSiH}^{-}$} & 2.09 & 2.05 & 2.05 & 2.05 & 2.11 & 2.07 & 2.07 & $1 \times 10^{-2}$ & \multirow{2}{*}{3.27} \\
\hline & 6.40 & 4.02 & 3.32 & 3.26 & 6.00 & 3.92 & 3.45 & $2 \times 10^{-2}$ & \\
\hline
\end{tabular}

${ }^{a}$ Oscillator strengths ( $f$ values) reported are for CCSD/d-aug-cc-pVTZ.

${ }^{b}$ Dunning's correlation consistent basis sets are abbreviated, e.g. t-aug-cc-pVDZ is tapVDZ. ${ }^{c}$ Computed with EOMIP-CCSD/t-aug-cc-pVDZ.

of these electronic transitions are within the wavelength range $(\sim 1-3.5 \mathrm{eV})$ of the DIBs and represent a new set of molecules whose relationship to the DIBs and the chemistry of the ISM have not been explored.

Closer examination of the difference in the excitation energies and the eBEs discloses that five of these anions have a substantial difference between the two values. Since dipole-bound states are Rydberg-like, the eBE and the dipole-bound excited state energy must be nearly coincident. This is observed for the two benchmarking anions. However, $\mathrm{CH}_{2} \mathrm{SiN}^{-}, \mathrm{SiCCN}^{-}, \mathrm{CCSiN}^{-}, \mathrm{C}_{3} \mathrm{H}^{-}$, and $\mathrm{CCSiH}^{-}$have differences of several tenths of an $\mathrm{eV}$. Vertical excitation energies are useful in classifying the nature of excited states since only the wavefunctions are manipulated. These are given in Table 2 for both the cc-pVDZ and cc-pVTZ families of increasingly diffuse basis sets. From this table it is clear that the first excited state for each of these anions is not dipole-bound since the majority of the spatial extent of the required basis set is described by the aug-cc-pVXZ basis. The large decline in the excitation energy as the basis sets become more diffuse for the higher-energy excitation clearly indicates the dipole-bound state. Additionally, these energies converge to within $0.1 \mathrm{eV}$ of the EOMIP vertical eBE. Furhermore, $\mathrm{CCSiN}^{-}$has two valence excited states below the eBE, the first small anion studied where such a result has been given. All of these anions with valence excitations contain silicon except $\mathrm{C}_{3} \mathrm{H}^{-}$. Although it is currently unclear as to why these anions possess valence excited states and others do not, especially for most of their first-row analogues, this phenomenon appears to be a combination of special $\pi \rightarrow \pi$ (not $\pi \rightarrow \pi^{*}$ ) excitations involving partially filled $\pi$-type orbitals as well as the charge separation brought about by the larger Si atom and its propensity only to form $\sigma$ bonds.

Multiple excited states of anions further gives new areas for exploration of potential DIB carriers. Conclusive assignments of excitation energies for any anions will probably result from laboratory experiments, but these theoretical computations have highlighted new physics that have been largely unexplored. Additionally, anions are attractive DIB carriers since most have only one excited state, and it is believed that most of the DIBs are not correlated. The excitation energies for most anions (especially those containing 
only the more astronomically abundant $\mathrm{H}, \mathrm{C}, \mathrm{O}$, and $\mathrm{N}$ ) are more toward the red end of the spectrum. The valence state of $\mathrm{C}_{3} \mathrm{H}^{-}$should be found at more than $13000 \AA$, for example. This reddening is consistent with a majority of the known DIBs and is further supported by new DIBs discovered by Geballe et al. (2011) beyond $10000 \AA$.

Excitation energies are useful, but detection of these anions in the ISM may first be necessary with vibrational or rotational spectroscopy. As such, the fundamental vibrational frequencies and spectroscopic constants of the DIB candidate $\mathrm{CH}_{2} \mathrm{CN}^{-}$are given in Fortenberry et al. (2013a). More excitingly, since the attribution of $\mathrm{C}_{3} \mathrm{H}^{+}$lines observed in the Horsehead nebula photodissociation region (PDR) has been questioned by Huang et al. (2013b), $1^{1} A^{\prime} \mathrm{C}_{3} \mathrm{H}^{-}$is the more likely candidate for those rotational lines from a spectroscopic perspective as suggested by Fortenberry et al. (2013b). With the existence of the valence state for this anion, it is likely that electron attachment rates in this region could be greater than the dissociation rate such that a steady state of $\mathrm{C}_{3} \mathrm{H}^{-}$ could be present. This would be the first small anion known to possess a valence excited state to be detected in the ISM.

\section{Acknowledgements}

Travel and research funds were provided to RCF by the NASA Postdoctoral Program administered through Oak Ridge Associated Universities. Prof. T. Daniel Crawford of Virginia Tech is acknowledged for his assistance with the excited state studies as well as for the use of the computing resources. Additionally, Drs. Xinchuan Huang and Timothy J. Lee of the SETI Institute and NASA Ames Research Center, respectively, were instrumental in the rovibrational studies undertaken.

\section{References}

Cordiner, M. A. \& Sarre, P. J. 2007, A\& A, 472, 537

Fermi, E. \& Teller, E. 1947, Phys. Rev., 72, 399

Fortenberry, R. C. 2013, Mol. Phys., in press (arXiv:1308.2916)

Fortenberry, R. C., \& Crawford, T. D. 2011a JPCA, 115, 8119

-. 2011b JChPh, 134, 154304

Fortenberry, R. C., Crawford, T. D., \& Lee, T. J. 2013a ApJ, 762, 121

Fortenberry, R. C., Huang, X., Crawford, T. D., Lee, T. J., 2013b, ApJ, 772, 39

Fortenberry, R. C., Huang, X., Francisco, J. S., Crawford, T. D., \& Lee, T. J. 2011 JChPh, 135, 134301

-. $2012 J C h P h, 136,234309$

Geballe, T. R., Najarro, F., Figer, D. F., Schlegelmilch, B. W., \& de la Fuente, D. 2011 Nature, 479,200

Gutsev, G. \& Adamowicz, A. 1995, ChPhL, 246, 245

Huang, X., Fortenberry, R. C., \& Lee, T. J. 2013a, JChPh, 139, 084313

-. 2013b, ApJL, 768, 25

Huang, X. \& Lee, T. J. 2008, JChPh, 129, 044312

Huang, X., Taylor, P. R., \& Lee, T. J. 2011, JPCA, 115, 5005

Lykke, K. R., Neumark, D. M., Andersen, T., Trapa, V. J., \& Lineberger, W. C. 1987, J. Chem. Phys., 87, 6842

McCall, B. J., Thorburn, J., Hobbs, L. M., Oka, T., \& York, D. G. 2001, ApJL, 559, 49

Mullin, A. S., Murray, K. K., Schulz, C. P., Szaflarski, D. M., \& Lineberger, W. C. 1992, Chem. Phys., 166, 207

Sarre, P. J. 2000, MNRAS, 313, L14

Simons, J. 2008, JPCA, 112, 6401

Tulej, M., Kirkwood, D. A., Pachkov, M., \& Maier, J. P. 1998, ApJL, 506, 69 